from $\mathrm{ku} \mathrm{v-s}$ or chu v-s, thus: ka, ke, ki, ko; ak, ek, ik, ok. It is an excellent example of how the same elements appear in new combinations with different phonetic values, these being influenced by vowel fluctuation.

Figs. $146,147=$ Uch v-s.

Fig. $148=\mathrm{Ka}$ v-s.

Figs. $149,150=$ Kan, ka v-s, an v-s.

Fig. ${ }_{5} \mathrm{I}=$ Yox, iax, yosh, iash, sh, h, xa v-s. The value of this element will be demonstrated in Part II. of this article.

The phonetic values assigned a series of elements having been given, let me proceed to apply some of them to certain glyphs beginning with Fig. 160 . This glyph, and variants of it, is frequently found in the codices in connection with a figure which has been designated, for the sake of convenience, "The Long-Nosed God," whom there is good reason to think is kukulkan. A glance at this glyph shows it to be a representation of an elongated reptile-like head, an ideographic suggestion of the serpent god. In the nose we have the elements, Fig. 105 $=$ cha, curved around into a loop-like end, Fig. 16r. The mouth line must not be confounded with the parallel earth line. At times small tooth-like squares (Figs. 28, 29, 30) are attached to it-similar to those shown in our Higs. $3 \mathbf{I}$ and 34 . They seem like phonetic additions placed to indicate the especial phonetic value of the element to which they are attached. In this case there are two squares attached $=\mathrm{ca}$. As chi = "mout" we accept the suggestion as cha or kha' (c $=\mathrm{k}$ in Maya). It will be observed that the end of the mouth line is somewhat curved upward (see Fig. 162). It might at first be thought the result of accident but an examination of other glyphs (Figs. I 7 I and $18 \mathrm{r}$ ) shows that this is not the case. Figs. 18r, r86, 187, show the mouth element, Fig. 185, connected with a curved line, a motive derived from the life line of the serpent kan, Fig. 192. This line, Fig. 187 a and Fig. I33 has the phonetic value of kan, ka v-s, an v-s. Chan is the evident phonetic value represented by our element, Fig. 162. We shail see it repeated with like value in other face glyphs yet to be analyzed. Fig. I63 we have assigned the phonetic value of $\mathrm{uch} \mathrm{v}-\mathrm{s}$, and by vowel fluctuation we obtain cha (see values assigned Figs. 146, 147). The element Fig. $167=$ cha or kha or ka. The element shown in Fig. $\mathrm{r}_{5}$ is composed of the perpendicular line, Fig. $\mathbf{I}=\mathrm{ka}$, and the twisted line, Fig. I 35 and Fig. I $53^{\mathrm{a}}=$ ban v-s, ba v-s an, $b$; its value an is here used, which, placed after $\mathrm{ka},=$ kan or kaan. Fig. 165 by reference to the list at Fig. $45=0$ or $u$. Fig. $166=$ cha; it is a variant of the ch'i glyph, Figs. 92 and 93, I 14,115, I 16, I 17 . The Fig. I 68 has a like value, as our element Fig. $99=$ chan or kan. The components, Figs. 169 , I 70 , I $70^{a}$, by reference to Figs. 7, 8, will be seen to have the value of $\mathrm{ka}$ or ca. All of the elements composing this glyph are kan elements recalling "chu-cha-chan" or kukakan.

Fig. I 7 I from the Codex Cortesianus is composed of a similar series of kan elements, the three perpendicular black dots to the right of it repeating xo (=three) or chu; so is Fig. 181 with its components, Figs. I82, $183,184,185,187$; all kan elements arranged into a face glyph.

In Fig. I7z we have another important face glyph which is a composite of kan elements. Curving upward around the muth ( $=$ ch'i) Fig. I $72^{\circ}$ is the an curve, Fig. 84, recalling chan. The element in the nose position, Fig. 173, = cha; see Figs. 109, 110, 111. The curved line, Fig. 174, = cha. It is a variant of Figs. 87 and 88 of my list and appears in many different combinations with this value. Some of them will be demonstrated in Part II. of this article. Fig. 175 bas already been used, as cha v-s in Fig. 160. The same phonetic value is represented here. Fig. 176 is a series of Figs. I and $2=$ $\mathrm{ka}$, and Fig. 177 are variants of Figs. 12 and $\mathrm{I} 3, \mathrm{I}_{4}=$ an, giving kan. All the elements in this face gli ph are kan elements. Where the dotted line Fig. $\mathbf{I}_{72^{\mathrm{a}}}$ is prefixed to the glyph it gives the hissing sound of $\mathrm{x}$, sh, or ch and the glyph becomes xan or chan.

Fig. I 78 has as one of its components a variant of the kan glyph, Fig. 172. The face or head is represented in the act of sucking the nipple of a breast. Hoobnelil, Fig. I 79. Inside of the outline of the breast, Fig. I 79, is the ah prefix, Fig. 180 . We have thus recalled by the prefix ah, by the representation of a breast, hoobnil, and by the glyph, a variant of Fig. 172, = kan, the name of the bacab or chak, who represents the cardinal point south, = ah-Hoobnil-kan. The glyph is taken from the series, Codex Troano, Plate $25^{*}$, and proves the assignment made by De Rosny to be correct. It is an excellent example of the ikonomatic method of writing used by the ancient Mayas, a similar method being used by the ancient Mexicans, and to use the words of Dr. D. G. Brinton in a letter received by me from that distinguished Americanist, "hence it proba: bly obtained in the Maya."

[To be continued.]

\section{DOUBLE SURFACES.}

\section{BY HENRY I. COAR, CAMBRIDGE, MASS.}

Tre double surface was discovered by Moebius, probably about 1858, and he called attention to some of the peculiarities of the surface as he constructed it, and which has been called after his name, "Blatt des Móebius." Since his time this surface has been studied to some extent, especially by German mathematicians, and many forms of the double surface have been found beside that of Moebius. The most recent work on the subject is by $F$. Dingledey"Topologische Studien über die aus ringförmig geschlossenen Bändern durch gewisse Schnitte erzeugbaren Gebilde" (Leipzig, 1890). In this work Dingledey gives a pretty complete bibliography of the subject. The existence of these surfaces is, however, little known, and it may be of interest to describe the simplest form, aside from any mathematical interest which may be attached to the subject.

The simplest form of a double surface may be constructed as follows: Take a strip of paper, whose edges we will denote as in the figure by $A B$ and $C D$, and bend it imto a ring, at the same time revolving one end through $180^{\circ}$, so that $B$ will fall on $C$ and $D$ fall on A. Now glue

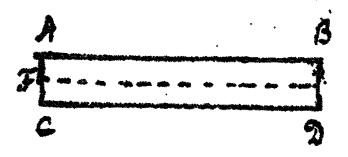

the two ends together. We shall then obtain a band, which has the distinctive properties that it is bounded by only one edge and has only one surface. In other words, we can pass from any point in the surface of the paper to the corresponding point on the other side of the paper without crossing the edge. This is the simplest form of a double surface.

If, now, we cut our band along the line marked EF in the figure, it will drop apart into a new band of twice the length of the former band, but the new band will no longer be a double surface. The reason for this is obvious. 
In the original band we could pass continuously along the edge from $\mathrm{B}$ to $\mathrm{A}(=\mathrm{D})$ to $\mathrm{C}(=\mathrm{B})$ back to our starting point. Now, in cutting along the line EF we nowhere cross this continuous edge, so that it will remain an edge of the new surface, while the cut will form a second edge of the new surface. We have thus removed one of the distinctive characteristics of the double surface, by having a surface bounded by two lines. Furthermore, it will be impossible to pass in the new band from a point on one side of the paper to the corresponding point on the opposite side without crossing the edge. The reason for this will be more obvious in the following:

In forming the double service we revolved one end of the strip of paper through an angle of $180^{\circ}$, about, say, the point $\mathrm{E}$, or, better, the line EF. It is clear now that if, instead of revolving it through an angle of $180^{\circ}$, we had revolved it through an angle of $2 \times 180^{\circ}$, the point $B$ would have fallen on $A$ and $D$ on $C$, so that we should have two continuous edges to our surface, and it will no longer be a double surface. We can then say, in general, that if we revolve the eud of the strip of paper through an odd multiple of $180^{\circ}$, before fastening the two ends together, we shall always obtain a double surface, whereas if we revolve through an even multiple of $180^{\circ}$ we have an ordinary surface. By this means we can distinguish double surfaces from ordinary surfaces.

We will now return to the surface obtained by cutting the double surface along the line EF and see why it must be an ordinary surface. The double surface originally had a twist of $180^{\circ}$. Suppose, now, we have cut it as indicated, but do not let the ends drop apart; then each part on either side of the cut will have a twist of $180^{\circ}$, or, together, $2 \times 180^{\circ}$. If we let the surface fall apart we double the twists again, and our new surface has a twist of $4 \times 180^{\circ}$, and it is therefore an ordinary surface. That this is so may be easily verified by cutting the band across and revolving one end until the strip has no twists, when it will be found that it has to be revolved through $4 \times 180^{\circ}$.

If we cut a double surface obtained by rotating through $3 \times 180^{\circ}$ along the line EF, we shall find that we have introduced a knot in our new surface, which in other respects will, however, be an ordinary surface. These knots will be multiplied, as we proceed, to surfaces containing a higher number of twists.

It is easy to see that if we cut an ordinary surface, obtained by revolving the end of our paper through $2 \times 180^{\circ}$, along the line EF, we shall obtain two ordinary surfaces, which are, however, interlinked. The same holds for surfaces with a higher number of twists, where, however, the interlinking becomes more complicated.

Another interesting set of results may be obtained by cutting the surfaces along a line parallel to the edge at a distance from the edge less than one-half the width of the strip of paper. The results will be different in the case of double and ordinary surfaces.

\section{BOTANICAL NOTES FROM WESTERN PENN- SYLVANIA.}

By hUBERT LYMAN CLARK, PITTSBURGH, PA.

ON looking over. my field notes for the spring and summer of 1893 , I find there are a few facts the preservation of which may be worth while, in the hope that before long some competent botanist will prepare an annotated list of the plants of western Pennsylvania. There is not at present, so far as I know, any such list, and its appearance would be welcomed by all our local botanists. Whenever the work is undertaken it will be desirable to have as much material in available form as possible, and so $t$ have presumed to publish my im. portant notes in Science, hoping they will also prove of interest to botanists elsewhere.

On analyzing specimens of Delphinium from the country around Pittsburgh last spring, I was struck with the rich coloring of the flowers. There was not the least doubt about the plant being $D$. tricome, but, to my surprise, Gray's "Manual" says the flowers of $D$. tricome are "bright-blue, sometimes white," while every specimen which I examined had "royal purple" flowers. Thinking that the trouble might be in my sense of color, I looked through the "Manual" for other "bright-blue" flowers. I found Aster undulatus, Chicorium intybus and Campanula rotundifolia so given, and I should certainly call them so, but the Delphinium of this vicinity has flowers of the same color as Liatris scariosa, which Gray calls "rose-purple," or perhaps nearer to Aster novoeanglice, which is given as "violet-purple." Never having seen Delphinium growing elsewhere, I am curious to know if in other parts of its range it really does bear flowers similar in color to Chic.rium or Campanula, or whether it is not a slip of the pen in the "Manual" to describe them as "bright-blue." In the same work (which is perfectly invaluable to an amateur botanist in the east) Silene nivea is recorded as "rare," and it is with great pleasure, therefore, that I can report it as abundant in several places around Pittsburgh. Indeed, I am inclined to think it is the most common representative of its genus in this neighborhood.

None of the botanists whom I have consulted record Trifolium stoloniferum east of Ohio, and it is therefore very pleasant to be able to record it from Pittsburgh. On the 8th of last June I found it growing in an open space in some woods about six miles east of the city. While it is of course possible that it has been introduced, it was growing so far from any house or highway as to certainly appear indigenous. There is no specimen of this clover in the herbarium of the Western Pennsylvania Botanical Society, and I am inclined to think this is the first record for the State.

Gnaphalium purpureum is reported in the "Manual" to occur in "sandy or gravelly soil, coast of Maine to Virginia and southward." It is not very clear from this how far inland we may expect to find it, but certainly the implication is that it is a seashore plant It may be worth while, therefore, to record that it is not very rare around Pittsburgh, three hundred and fifty miles from the coast! I found it growing in Arlington, twelve miles south of the city, in June last, and there are a number of specimens in the herbarium of the botanical society to which reference has been made. These specimens are from widely-scattered points in the county, and would seem to indicate regular and not uncommon occurrence. One of the most abundant weeds in many parts of Pittsburgh is a species of Galinsoga, differing from $G$. parviflora in the scales of the pappus and being very hairy instead of smoothish. Dr. Robinson, of the Gray Herbarium, Cambridge, to whom I am indebted for many favors, kindly identified the specimens sent to him as "a possible variety of $G$. hispida," or at least so it may be considered provisionally. Similar specimens are reported from Milwaukee and Providence. Whether G. parviflora occurs in Pitts burgh I cannot say, but I have not yet found any specimens agreeing with the description in the "Manual." Another plant which Dr. Robinson identified for me is also an introduced species reported in the "Manual" as "rather rare; in cultivated grounds." I refer to Veronica arvensis, two specimens of which I sent to Cambridge, supposing them to represent different species, they were so unlike. One of them was collected in open pasture land, and I found similar specimens in 